\title{
2D Data-Driven Stalk Cell Prediction Model Based on Tip-Stalk Cell Interaction in Angiogenesis
}

\author{
Mengmeng Wang ${ }^{1}$, Lee-Ling Sharon Ong ${ }^{2}$, Justin Dauwels ${ }^{1}$, H. Harry Asada ${ }^{2,3}$ \\ ${ }^{1}$ School of Electrical and Electronic Engineering, Nanyang Technological University, Singapore. \\ ${ }^{2}$ Singapore-MIT Alliance for Research and Technology, Singapore. \\ ${ }^{3}$ Department of Mechanical Engineering, MIT Cambridge, MA, USA. \\ email: mwang009@e.ntu.edu.sg and jdauwels@ntu.edu.sg
}

\begin{abstract}
Angiogenesis is the growth process of blood vessels from existing vessels. During angiogenesis, endothelial cells (ECs), which line the vessel, specialize into tip and stalk cells. Tip cells respond to angiogenic signals, burrow into the extracellular matrix (ECM) and form conduits. Stalk cells follow the tip cells along the conduits, and form solid sprouts or lumen vessels. Interactions between stalk cells and tip cells are important for creating functional vessels. The goal of this work is to predict stalk cells migration trajectories from known tip cell trajectories. Four factors influence the position and velocity of cell migration in ECM: cell-cell interaction, drag force, chemotactic signal and cell-ECM interaction. As chemotactic signal and cell-ECM interactions have little effect on stalk cell movement, the proposed model includes the influence of cell-cell interactions and drag force only. The unknown parameters in the model are inferred by Maximum Likelihood Estimation (MLE) from experimental time-lapse cell migration data. Numerical results suggest that the proposed model can accurately predict stalk cell trajectories. The proposed model may be useful for the study of angiogenesis, a critical process for cancer tumor growth.
\end{abstract}

\section{INTRODUCTION}

Cancer is a group of diseases that have a common feature of uncontrolled cellular growth, and causes $13 \%$ of all human deaths worldwide [1]. Angiogenesis, which is the growth process of blood vessels from existing vessels, is critical for cancer invasion [2]. Tumor-induced angiogenesis starts with the activation of Endothelial Cells (ECs) by Tumor Angiogenic Growth Factors (TAFs), followed by migration and proliferation and finally vessel maturation [3]. ECs specialize into tip, stalk and phalanx cells in angiogenesis [4]. Tip cells sense and respond to the guidance cues, burrow into the extracellular matrix (ECM) and form conduits. Stalk cells trail behind tip cells along the conduits and form solid sprouts or lumen vessels.

In related work, the angiogenesis process is modeled using continuous and discrete mathematical approaches [1]. Continuous models use Partial Differential Equations (PDEs) to reflect the distribution of ECs and angiogenic factors. While discrete approaches model these factors individually. Anderson and Chaplain assumed that the growth of a single vessel is governed by its tip cell movement and this tip cell moves across a rectangular network according to pre-defined rules [5]. At each step of the simulation, the probability that a tip cell moves in one of four specified directions was calculated using the continuous approach. This model does not fully represent cell trajectory because cells can only move in the specified directions in the network. Wood et al. developed an extended agent-field model to predict EC behavior in angiogenesis [6]. In this model, the stochastic behavior of ECs was modeled as a population of stochastic agents, each having a Markov chain state transition graph. The state transition probabilities vary depending on matrix cues such as growth factors and matrix gel properties.

Our work is novel in two ways: First, our prediction model uses only Ordinary Differential Equations (ODEs), reducing the modeling complexity. Second, the unknown parameters are tuned using cell trajectories obtained from automated image analysis of experimental observations. Our data-driven model is able to predict stalk cell trajectories accurately with low computational requirements and is easily scalable for large cell populations.

This paper is structured as follows. In Section II, we provide background on the influencing factors of position and velocity of the ECs. In Section III, we explain our mathematical model, and in Section IV we provide numerical results. In Section V, we offer concluding remarks.

\section{BACKGROUND}

There are four factors that may influence the position and velocity of the ECs. They are cell-cell interaction, drag force, chemotactic signal and cell-ECM Interaction [7].

\section{A. Cell-Cell Interaction}

ECs can interact with their neighbours through adhesive and repulsive forces. These two forces are modeled based on work in [7].

1) Cell-Cell Adhesion: Cell adhesion, which is regulated by cell-adhesion molecules (CAMs) expressed at the cell surface, is essential for many cell physiological functions (e.g. proliferation, differentiation and migration) and pathological conditions (e.g. inflammation and interaction of cell with biomaterial) [8]. From [7], the adhesion force on cell $i$ impacted by cell $j$ is:

$$
\begin{aligned}
\mathbf{F}_{c c a}^{i j} & =-c_{c c a} \nabla \varphi\left(r ; R_{A} ; n\right) \\
& =\left\{\begin{array}{ll}
-c_{c c a}\left(1-\frac{|r|}{R_{A}}\right)^{n+1} \frac{r}{|r|} & 0<|r| \leq R_{A} \\
0 & |r|>R_{A}
\end{array},\right.
\end{aligned}
$$


where $c_{c c a}$ is the adhesion constant, $r=\mathbf{x}^{j}-\mathbf{x}^{i},|r|$ is the Euclidean norm of $r, R_{A}$ is the maximum adhesive interaction distance, and $\mathrm{n}$ is the exponent of the potential.

2) Cell-Cell Repulsion: As cells can mechanically resist compression, a repulsive force is introduced when two cells come into contact with each other. This repulsive force increases rapidly when these two cells move closer, especially when their nuclei are in close proximity. The repulsive force is computed as [7]:

$$
\begin{aligned}
\mathbf{F}_{c c r}^{i j} & =-c_{c c r} \nabla \psi\left(r ; R_{N} ; R ; M ; m\right) \\
& =\left\{\begin{array}{ll}
c_{c c r}\left(c \frac{|r|}{R_{N}}+M\right) \frac{r}{|r|} & 0<|r|<R_{N} \\
c_{c c r}\left(1-\frac{|r|}{R}\right)^{m+1} \frac{r}{|r|} & R_{N} \leq|r| \leq R \\
0 & |r|>R
\end{array},\right.
\end{aligned}
$$

where $c_{c c r}$ is the repulsive constant, $r=\mathbf{x}^{j}-\mathbf{x}^{i},|r|$ is the Euclidean norm of $r, R_{N}$ is cell nucleus radius, $R$ is cell radius, $M \geq 1$ is the cells' maximum repulsive force, $m$ is a fixed nonnegative integer, and $c=\left(1-\frac{R_{N}}{R}\right)^{m+1}-M$.

\section{B. Drag Force}

Cells experience drag forces during their motion in ECM. For a very low Reynolds number (e.g. $R e<1$ ), the drag force is approximately proportional to velocity, in the opposing direction [9]. As the cell velocity is very small and the Reynolds number is proportional to its velocity, the drag force on cell $i$ can be calculated as:

$$
\mathbf{F}_{\text {drag }}^{i}=-v \dot{\mathbf{x}}^{i}
$$

where $v$ is the viscosity of ECM and $\dot{\mathbf{x}}^{i}$ is velocity of cell $i$.

\section{Chemotactic Signal}

When the concentration of VEGF and other angiogenesis factors around the cell $i$ exceeds a threshold value, the filopodia of the tip cells will sense the signals, leading to cell migration due to chemotactic forces [1] [4].

\section{Cell-ECM Interaction}

During angiogenesis, tip cells secrete Matrix Metalloproteinases (MMPs) to degrade the ECM and create conduits for stalk cell migration [1]. Tip cells also interact with the ECM during the movement, hence we regard ECM as a spring with an elastic constant $k_{E C M}$ [7].

As shown in earlier studies [10], tip cells sense the chemotactic signals in the microenvironment, respond accordingly through their filopodia, and create conduits. On the other hand, stalk cells follow behind the tip cells in the conduits created. Cell-ECM interactions are more prominent in tip cells [1] [7]. Therefore, we do not account for the influence of chemotactic signals and cell-ECM interactions when predicting stalk cell trajectories in our model.

\section{Mathematical Modeling}

\section{A. Governing Function}

We define the position and velocity of the ith cell at time $k$ as:

$$
\mathbf{x}_{k}^{i}=\left[\begin{array}{c}
x_{k}^{i} \\
y_{k}^{i}
\end{array}\right] \in \mathbb{R}^{2}, \quad \quad \mathbf{v}_{k}^{i}=\left[\begin{array}{c}
v_{x k}^{i} \\
v_{x k}^{i}
\end{array}\right] \in \mathbb{R}^{2} .
$$

Newton's Second Law states that the acceleration a of a body is proportional to the net force $\mathbf{F}$ acting on its body and inversely proportional to its body mass $m$. As acceleration $\mathbf{a}$ is the first order derivative of velocity and second order derivative of position, the net force on the ith cell can be represented as:

$$
\mathbf{F}^{i}=m \mathbf{a}^{i}=m \frac{d \mathbf{v}^{i}}{d t}=m \frac{d^{2} \mathbf{x}^{i}}{d t^{2}} .
$$

The net force exerted on ith cell consists of cell-cell interactions and drag force, as explained in section II. The governing function of these forces on cell $i$ is described as:

$$
\begin{aligned}
\mathbf{F}^{i} & =\mathbf{F}_{c e l l-c e l l}^{i}+\mathbf{F}_{d r a g}^{i} \\
& =\sum_{j=1, j \neq i}^{N}\left(\mathbf{F}_{c c a}^{i j}+\mathbf{F}_{c c r}^{i j}\right)+\mathbf{F}_{d r a g}^{i},
\end{aligned}
$$

where $j=1,2, \ldots, N$, and $j \neq i$ represents all cells in the experiment except cell $i$.

\section{B. Prediction Equation}

The second order ODE for $\mathbf{x}$ in equation (4) can be split into two first order ODEs:

$$
\frac{d \mathbf{x}^{i}}{d t}=\mathbf{v}^{i}, \quad \frac{d \mathbf{v}^{i}}{d t}=\frac{\mathbf{F}^{i}}{m} .
$$

The first order ODEs can be solved numerically by Euler's forward iteration, resulting in (8) and (9). The variables $\hat{\mathbf{x}}$ and $\hat{\mathbf{v}}$ represent the predictions of cell position $\mathbf{x}$ and cell velocity $\mathbf{v}$ respectively.

\section{Parameter Estimation}

1) Assumption: To numerically solve equation (7), we assume:

- All cells have the same mass,

- All cells are round,

- The cell diameter is 2.5 times large of its nucleus diameter as the nucleus diameter can be obtained from experiments.

2) Initial Condition: In our model, we use the experimental data at $k=1$ as the initial condition.

3) Known Parameters: Several parameters in equation (9) are obtained from the literature [11]:

- The exponent of the potential for adhesion: $n=1$,

- Maximum adhesive interaction distance: $R_{A}=1.214 R$,

- The exponent of the potential for repulsion: $m=1$,

- Cell maximum repulsive force: $M=1$.

4) Unknown Parameters: Three unknown parameters need to be estimated in equation (9):

$$
a=\frac{v}{m}, \quad b=\frac{c_{c c r}}{m}, \quad \text { and } \quad d=\frac{c_{c c a}}{m} .
$$

The prediction error $\varepsilon_{k}$, defined as the discrepancy from the actual experimental measurements $\mathbf{v}_{k}^{i}$, depends on those three parameters:

$$
\varepsilon_{k}(a, b, d)=\mathbf{v}_{k}^{i}-\hat{\mathbf{v}}_{k}^{i}
$$




$$
\begin{aligned}
& \hat{\mathbf{x}}_{k+1}^{i}=\hat{\mathbf{x}}_{k}^{i}+\hat{\mathbf{v}}_{k}^{i} \Delta t . \\
& \hat{\mathbf{v}}_{k+1}^{i}=\left(1-\frac{v}{m} \Delta t\right) \hat{\mathbf{v}}_{k}^{i}+ \begin{cases}\left(\frac{c_{c c r}}{m}\left(c \frac{|r|}{R_{N}}+M\right)-\frac{c_{c c a}}{m}\left(1-\frac{|r|}{R_{A}}\right)^{n+1}\right) \frac{r}{|r|} \Delta t & 0<|r|<R_{N} \\
\left(\frac{c_{c c r}}{m}\left(1-\frac{|r|}{R}\right)^{m+1}-\frac{c_{c c a}}{m}\left(1-\frac{|r|}{R_{A}}\right)^{n+1}\right) \frac{r}{|r|} \Delta t & R_{N} \leq|r| \leq R \\
-\frac{c_{c c a}}{m}\left(1-\frac{|r|}{R_{A}}\right)^{n+1} \frac{r}{|r|} \Delta t & R<|r| \leq R_{A} \\
0 & |r|>R_{A}\end{cases}
\end{aligned}
$$

For the sake of convenience, we make the common assumption that the prediction error $\varepsilon$ is Gaussian with zero mean:

$$
\varepsilon_{k}(a, b, d) \sim N(0, S),
$$

where $S$ is the noise covariance. If the covariance $S$ is isotropic, we have:

$$
S=\left[\begin{array}{cc}
\sigma^{2} & 0 \\
0 & \sigma^{2}
\end{array}\right],
$$

and the unknown parameter vector becomes:

$$
\theta=\left[\begin{array}{llll}
a & b & d & \sigma
\end{array}\right]^{T} .
$$

The Maximum Likelihood Estimate (MLE) of the parameter vector based on the experimental data from $k=1$ to $T_{f}$ is given by:

$$
\begin{aligned}
\hat{\theta}^{M L E} & =\arg \max _{\theta} \prod_{k=1}^{T_{f}}\left(\frac{1}{2 \pi|S|^{\frac{1}{2}}} \exp \left(-\frac{1}{2} \varepsilon_{k}(\theta)^{T} S^{-1} \varepsilon_{k}(\theta)\right)\right) \\
& \left.=\arg \min _{\theta} \sum_{k=1}^{T_{f}}\left(\varepsilon_{k}(\theta)^{T} S^{-1} \varepsilon_{k}(\theta)\right)+\ln |S|\right)
\end{aligned}
$$

Our experimental data contains trajectories of cells migrating alone, in addition to trajectories of stalk cells following tip cells. To limit the computational complexity, we estimate the parameters in two steps. First, the parameters $a$ and $\sigma$ are inferred from experimental trajectories of cells migrating alone, where only the drag force and random noise is included in the model. Next, the remaining parameters $b$ and $d$ in (9) are estimated from experimental trajectories of stalk cells following tip cells.

\section{RESUlTS AND DISCUSSION}

Our angiogenic experiments are performed using "high throughput" microfluidic devices designed by Farahat et al. [12] (see in Fig. 1). Collagen gel is injected in channel C. Vascular Endothelial Growth Factors (VEGFs) play an important role in angiogenesis, since cells tend to migrate towards a higher gradient of VEGF. To create a VEGF concentration gradient across the gel, we provide a higher VEGF concentration to channel A than to channel B. The sprouting process is observed by means of a confocal microscope at 30-minute intervals. We extract cell trajectories from the acquired images using automated image processing algorithms.

From the resulting trajectories, we infer the ML estimates of the parameters in (8) and (9). Next we predict the stalk cell trajectories from the tip cell trajectories. As an

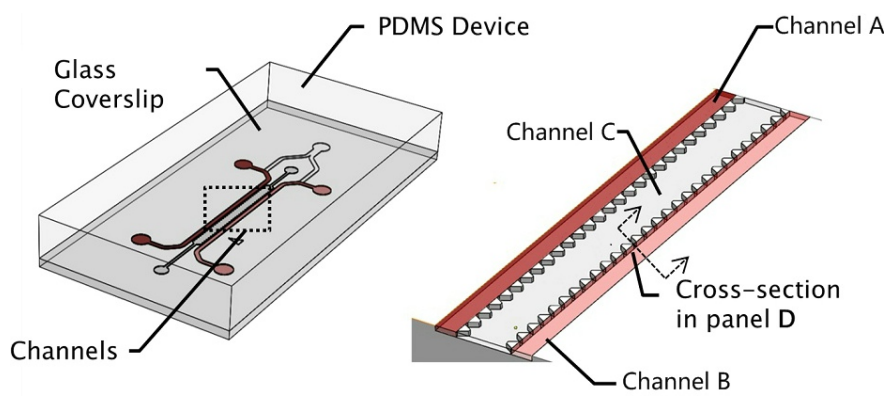

Fig. 1. "High throughput" microfluidic device [12].

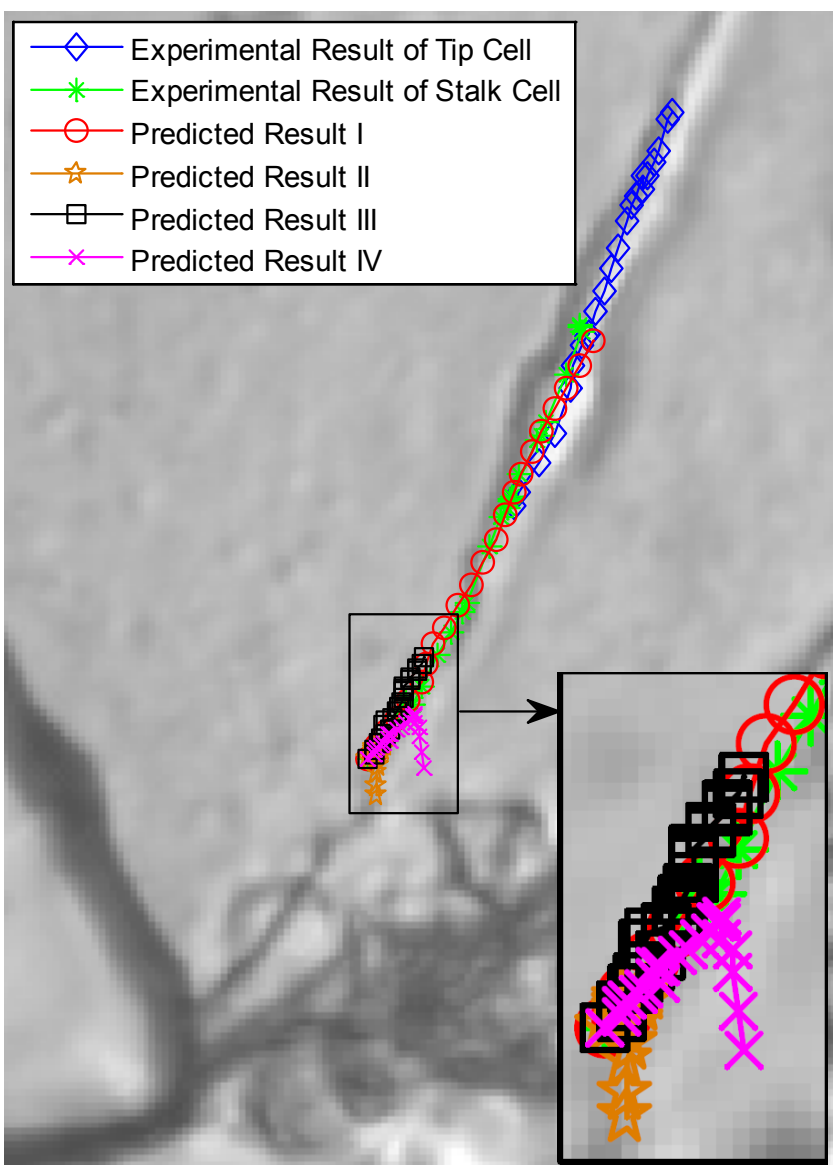

Fig. 2. Comparison of the Experimental and Predicted Stalk Cell Trajectory.

illustration, Fig. 2 shows our results for one stalk and tip cell trajectory. We consider four different ways (I to IV) to predict the stalk cell trajectory. In the first approach, 
we apply our model (8) and (9), which includes both cellcell interactions and drag force. In the second approach, we neglect the cell-cell interaction and only consider the influence of drag force; in the third approach, only cell-cell interactions are considered. At last, in the fourth approach, the stalk cell moves randomly in ECM by neglecting all the force exerted on it. The background of Fig. 2 is the final image acquired by the confocal microscope during that particular experiment. As can be seen from Fig. 2, our model yields the most accurate stalk trajectories (predicted results I). This observation implies that both cell-cell interactions and drag force are critical to predict talk cell trajectories.

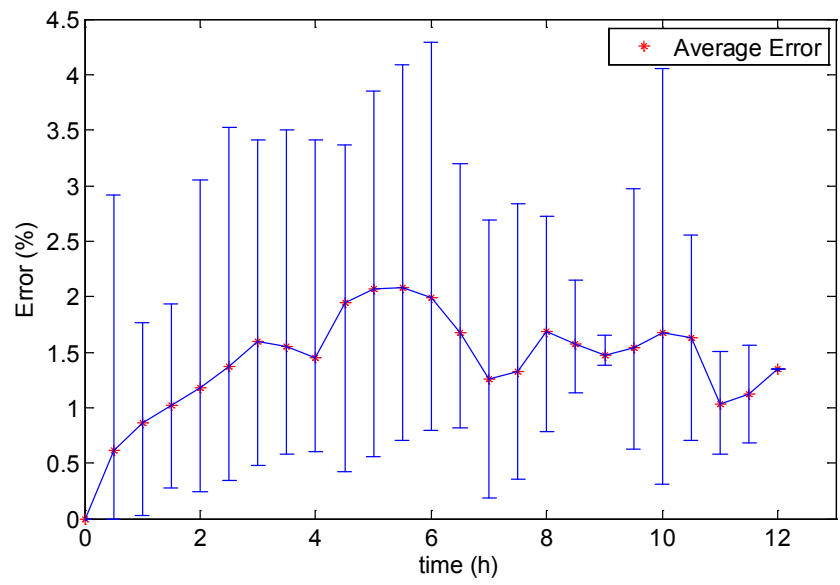

Fig. 3. Error from Predicted Result I and Experimental Result of Stalk Cell Trajectory over Time.

Fig. 3 shows the average prediction error and standard deviation for model (8)(9), averaged over ten stalk cell trajectories. From that figure, it can be seen that our model is able to consistently predict the stalk cell trajectory with given tip cell information, as the deviation is less than $5 \%$ and does not increase with time.

There are several possible sources for this deviation. First, we assume that the cell is round and the cell radius is 2.5 times its nucleus radius, which may not be the case in real experiments. Second, the cell migrates in 3D but the relative distances between the tip and stalk cells were computed in 2D. Our model does not account for any distances in the third direction, which may contribute to the error.

\section{CONCLUSION}

In this paper, we have proposed a data-driven mathematical model to predict stalk cells migration from known tip cell trajectories. As chemotactic signal and cell-ECM interactions have little effect on stalk cell movement, the proposed model includes the influence of cell-cell interactions and drag force only. The computational complexity of the model is low, since it only contains ordinary differential equations and is free of partial differential equations. The model predicts stalk cell trajectories accurately, yet it is substantially simpler than existing models.

In future work, we will extend our model to include angiogenic factors such as growth factor concentration and cell shape. In addition, we will analyze more experimental data.

\section{ACKNOWLEDGEMENT}

This research was supported by the National Research Foundation Singapore through the Singapore MIT Alliance for Research and Technology's BioSystems and Micromechanics Inter-Disciplinary Research programme. The authors would like to thank Soheila Sharghi Namini and Even Tan for providing experimental data.

\section{REFERENCES}

[1] N. Mantzaris, S. Webb, and H. Othmer, "Mathematical modeling of tumor-induced angiogenesis," Journal of mathematical biology, vol. 49, no. 2, pp. 111-187, 2004.

[2] H. Gerhardt, "Vegf and endothelial guidance in angiogenic sprouting," VEGF in Development, pp. 68-78, 2008.

[3] A. Das, D. Lauffenburger, H. Asada, and R. Kamm, "A hybrid continuum-discrete modelling approach to predict and control angiogenesis: analysis of combinatorial growth factor and matrix effects on vessel-sprouting morphology," Philosophical Transactions of the Royal Society A: Mathematical, Physical and Engineering Sciences, vol. 368, no. 1921, pp. 2937-2960, 2010.

[4] F. De Smet, I. Segura, K. De Bock, P. Hohensinner, and P. Carmeliet, "Mechanisms of vessel branching filopodia on endothelial tip cells lead the way," Arteriosclerosis, thrombosis, and vascular biology, vol. 29, no. 5, pp. 639-649, 2009.

[5] A. Anderson and M. Chaplain, "Continuous and discrete mathematical models of tumor-induced angiogenesis," Bulletin of mathematical biology, vol. 60, no. 5, pp. 857-899, 1998.

[6] L. Wood, R. Kamm, and H. Asada, "Stochastic modeling and identification of emergent behaviors of an endothelial cell population in angiogenic pattern formation," The International Journal of Robotics Research, vol. 30, no. 6, pp. 659-677, 2011.

[7] G. D'Antonio, P. Macklin, and L. Preziosi, "An agent-based model for elasto-plastic mechanical interactions between cells, basement membrane and extracellular matrix," Math. Biosci. Eng, 2012.

[8] C. Verdier, J. Etienne, A. Duperray, and L. Preziosi, "Review: Rheological properties of biological materials," Comptes Rendus Physique, vol. 10, no. 8, pp. 790-811, 2009.

[9] G. Batchelor, An introduction to fluid dynamics. Cambridge university press, 2000 .

[10] M. Hellström, L. Phng, J. Hofmann, E. Wallgard, L. Coultas, P. Lindblom, J. Alva, A. Nilsson, L. Karlsson, N. Gaiano, et al., "Dll4 signalling through notch1 regulates formation of tip cells during angiogenesis," Nature, vol. 445, no. 7129, pp. 776-780, 2007.

[11] P. Macklin, M. E. Edgerton, A. Thompson, and V. Cristini, "Patientcalibrated agent-based modelling of ductal carcinoma in situ (DCIS): From microscopic measurements to macroscopic predictions of clinical progression," J. Theor. Biol., 2012. (in press).

[12] W. Farahat, L. Wood, I. Zervantonakis, A. Schor, S. Ong, D. Neal, R. Kamm, and H. Asada, "Ensemble analysis of angiogenic growth in three-dimensional microfluidic cell cultures," PloS one, vol. 7, no. 5, p. e37333, 2012. 